\title{
Spécificités du salariat permanent en élevage laitier de montagne : une première approche dans les Alpes du Nord*
}

\section{Lucie Dupré}

Inra

Mona/SAE2

65, avenue de Brandebourg

94250 Ivry-sur-Seine

France

<dupre@ivry.inra.fr>

\begin{abstract}
Résumé
Le salariat fait une entrée discrète mais réelle dans les exploitations familiales d'élevage laitier de montagne d'où il était relativement absent jusqu'ici. Il s'ensuit des situations de travail définies, d'un côté par le cadre juridique du monde du travail en général et adossé au modèle industriel en particulier, et de l'autre, par les spécificités du travail agricole fondé sur la confiance et la familiarité propre à l'univers domestique. À partir d'enquêtes de terrain conduites en Savoie et en Haute-Savoie, l'étude propose une première approche de la spécificité du salariat permanent en pointant les tensions qui naissent de ce double régime de référence. Trois types de situations - salariat individuel, service de remplacement et groupement d'employeurs - sont analysés.
\end{abstract}

Mots clés : bovin laitier ; exploitation agricole familiale ; main d'œuvre permanente ; organisation du travail.

Thèmes : économie et développement rural ; productions animales.

\section{Abstract \\ Distinctive features of wage labour in family livestock farming: An initial approach based on a case study in the Northern Alps (France)}

Quite unusual in family livestock farming, notably in mountainous areas, wage labour in France is slowly but steadily gaining impetus. In so doing, it brings about work situations defined on the one hand by the legal framework characteristic of labour in the industrial world and on the other hand by the specifities of an agricultural world where transactions are governed by the trust and familiarity prevailing in family relationships. Based on field surveys carried out in Savoie and Haute-Savoie (French Northern Alps), the present study focuses on the specific features of permanent wage labour while pointing out to the tensions arising out of the two worlds it refers to. Three forms of legal frameworks are examined: individual full-time or part-time labour, wage labour within replacement services, and employment by a group of employers.

Key words: dairy cattle; family farms; permanent labour; work organization.

Subjects: animal productions; economy and rural development.

oulignant qu'en agriculture, le travail s'organise au sein d'un univers social avant tout familial, Barthez (1982) rappelle que "l'activité agricole n'est pas salariée". Ce propos garde une actualité certaine bien qu'il faille le nuancer. En effet, certains secteurs se sont développés grâce à une main-d'œuvre contractuelle importante et souvent saisonnière (cultures industrielles, viticulture, horticulture, maraîchage). Depuis une dizaine d'années, la tendance à l'agrandissement des élevages laitiers se conjugue avec le fait que les épouses

\footnotetext{
* Pour citer cet article: Spécificités du salariat permanent en élevage laitier de montagne: une première approche dans les Alpes du Nord. Cah Agric 2010; 19:366-70. DOI : 10.1684/ agr.2010.0423.
} 
occupent désormais souvent un emploi en dehors de l'exploitation. Cette situation se traduit par une augmentation de la charge de travail des éleveurs, alors que, dans le même temps, ceux-ci aspirent à de meilleures conditions de vie ce à quoi le salariat permanent peut contribuer. Ce dernier renvoie à la catégorie proposée par l'Institut national de la statistique et des études économiques (Insee) désignant "la main-d'œuvre non familiale occupant un emploi sur l'exploitation pendant toute la campagne agricole». En élevage laitier, il est plutôt masculin, jeune et relativement permanent (Rattin, 2003).

Trois cadres juridiques principaux existent qui répondent à des besoins spécifiques en main-d'œuvre :

- l'emploi individuel à temps complet ou à temps partiel (le plus souvent le fait de groupements agricoles d'exploitation en commun (GAEC) ;

- le salariat au sein d'un service de remplacement (SR) lequel, moyennant adhésion, met un vacher à la disposition des agriculteurs en cas de besoin (accidents, maternité, formation) ;

- et les groupements d'employeurs (GE). Sur la base d'une enquête exploratoire en élevage laitier dans les Alpes du Nord, quelques éléments permettant de caractériser, en première approche, la singularité du salariat agricole dans un contexte d'élevage familial ont été réunis. L'analyse, menée dans le cadre du programme ANR-ADD Trans se fonde sur les trois entrées suivantes : le recrutement, l'affectation des tâches et du temps de travail et les situations d'emploi partagé. On part de l'hypothèse qu'une tension se fait jour entre les référentiels qui polarisent les situations de travail dans le salariat. D'un côté, la forme de l'emploi (le contrat de travail) s'adosse au modèle industriel et tend à aligner juridiquement le salarié sur les autres actifs. De l'autre, la nature et les conditions d'exercice de l'activité (tâches, rythmes, contraintes) tendent, sous certains aspects, à renvoyer à des "rapports familiaux de production" (Barthez, 1982).

\section{Démarche méthodologique}

L'enquête concerne deux petites régions agricoles du massif des Bauges (l'Albanais et le Cour des Bauges) dans les Alpes du
Nord (Savoie et Haute-Savoie). L'élevage bovin laitier y prédomine, avec des exploitations d'une trentaine de bêtes et une tendance à l'agrandissement à travers notamment des formes sociétaires. Le salariat y renvoie historiquement au personnage du garçon de ferme ou au saisonnier et son savoir-faire très spécifique (le fromager ou le vacher d'alpage). Le salariat permanent s'y développe depuis quelques années sous les différents statuts présentés ci-dessus. L'étude a porté sur 10 éleveurs faisant appel soit à un salarié permanent, soit à un groupement d'employeurs soit encore à un service de remplacement, et sur leurs salariés respectifs. Les 10 exploitations laitières enquêtées sont pour la plupart des GAEC "bovin lait ", avec parfois une diversification des activités (élevage caprin, transformation fromagère, vente directe, activités équestres). L'échantillonnage a par ailleurs été constitué dans le souci de couvrir une grande diversité de situations, en termes d'âge des salariés (de 21 à 46 ans, dont 9 hommes et une femme), de statut (4 salariés partagés en GE ou SR, mais tous employés sur des contrats à durée indéterminée et les autres permanents mais parfois à mi-temps), de projet professionnel (7 ont un projet d'installation, les autres sont des salariés de métier, l'un étant pluriactif et l'autre en service de remplacement). Qu'ils soient ou non issus du milieu agricole, tous ont fait des études agricoles. Les plus jeunes ont un niveau BTS $^{1}$ ("Analyse et conduite des systèmes d'exploitations " ou "Production animale"). Deux d'entre eux, qui ne sont pas enfants d'agriculteurs, sont diplômés du secondaire et, singularité intéressante, se sont spécialisés en machinisme $\left(\mathrm{CAP}^{2}\right.$ "Conduite de machine en exploitation agricole " et Bac professionnel " Maintenance de machines agricoles "). Les deux salariés plus âgés (39 et 46 ans) et ayant le plus d'ancienneté dans leur poste, sont issus du milieu agricole et ont un $\mathrm{BEPA}^{3}$. Le salariat est pour tous un vrai choix professionnel et/ou un choix de vie à part entière.

Les entretiens ont été conduits d'abord auprès des agriculteurs employeurs puis auprès de leurs salariés rencontrés souvent sur leur lieu de travail mais en l'absence de leurs employeurs. Pour les salariés, la grille d'enquête très directive

\footnotetext{
1 BTS : brevet de technicien supérieur. 2 CAP : certificat d'aptitude professionnelle. 3 BEPA: brevet d'aptitude professionnelle agricole.
}

visait à décrire le parcours professionnel et les modalités de recrutement de ces derniers, les tâches et les responsabilités qui leur sont confiées, ainsi que leurs conditions de travail. L'objectif était de dégager la place occupée dans l'exploitation agricole et les éléments fondateurs de l'identité professionnelle du salarié. Pour les employeurs, le guide d'entretien visait à comprendre les motivations et les réticences face au salariat, les pratiques de recrutement et la réorganisation du travail liée à l'embauche d'un salarié.

\section{Résultats}

\section{Des origines qualifiantes}

L'enquête montre que le recrutement des salariés permanents s'opère via les réseaux professionnels (chambres d'agriculture et presse spécialisée) mais aussi et avant tout via les réseaux locaux l'Agence nationale pour l'emploi (ANPE) n'intervenant pratiquement pas. Les offres d'emploi se caractérisent par une faible (voire une absence) d'exigences en termes de qualification, ce qui pose la question des critères qui président à leur recrutement. La familiarité du candidat avec le milieu agricole, local ou non et/ou sa familiarité avec le territoire local apparaissent déterminantes dans la mesure où elles accréditent sa " motivation " (souvent l'une des seules exigences requises) et gagent d'une certaine confiance. Ainsi, sans grande surprise dans ce milieu à forte reproduction sociale, être issu d'une famille agricole est une qualification à part entière. Dans les demandes d'emploi, la mention "fils d'agriculteur" est couramment utilisée comme telle. Une expérience en service de remplacement peut également convaincre les employeurs face à un candidat totalement étranger, tant ce type de salariat est réputé exigeant et difficile (Frappat, 2005). La proximité géographique et donc sociale renforce ces deux premiers critères. Suivant Maget (1955), on peut considérer que ce n'est plus le village mais le massif des Bauges dans son ensemble qui constitue une "étoile d'interconnaissance" dans la mesure où, permettant "continuité et permanence du champ d'identification réciproque", il offre les garanties nécessaires à l'employeur. Il n'est pas rare, comme cela a été observé ailleurs (Chabanet et al., 2000), que certains 
salariés soient pressentis par un employeur avant même d'avoir postulé. Le lien au pays ne signifie pas forcément d'y résider en permanence, l'important étant de pouvoir être situé dans un espace social contrôlé. C'est le cas de ce jeune homme qui n'est pas d'origine agricole et qui vit en région parisienne où il travaille dans un grand élevage. Il vient depuis son enfance régulièrement dans les Bauges chez ses grands-parents. À la première occasion, il a quitté son emploi pour s'installer dans les Bauges et devenir salarié de l'élevage voisin auquel il donnait un coup de main lors de ses séjours. Le massif constitue cependant un espace réduit et l'ajustement de l'offre à la demande y est parfois difficile, obligeant les employeurs à recruter au-delà de cette zone socialement maîtrisée. Dans ce cas, et lorsque le candidat "inconnu " a un projet d'installation, il peut faire son stage préparatoire dans l'exploitation dont il souhaite par la suite être salarié quelque temps, avant de devenir luimême chef d'exploitation. Cela permet aux deux parties, employeur et employé, de prendre le temps de s'assurer que chacun y trouve satisfaction.

\section{" Un métier un peu complet dans tout "}

La polyvalence réfère encore parfois à l'ancienne figure fortement stigmatisée de "l'homme toutes mains qualifié". Dans les années 1970, ce dernier est " capable de tous les travaux de la ferme et des champs et d'aider toutefois aux travaux courants de l'élevage et de la traite "Or, note Bourquelot (1973), " on est amené à déduire logiquement que ce "peut tout faire " est équivalent de "bon à rien ", ce qui, ajoute-t-elle, " est sans doute un classement facile". La situation a nettement évolué et dans les élevages enquêtés, la polyvalence est largement réhabilitée. Maintenant également désignée sous le terme "multicompétence " (Peltier et al., 2009) qui atténue cette image restée longtemps négative, elle est à la fois une exigence forte des employeurs et une revendication des salariés.

Les salariés apprécient et valorisent la polyvalence parce qu'elle renvoie aux réalités d'une identité professionnelle à laquelle, souvent, ils aspirent, à savoir le statut de chef d'exploitation (Frappat, 2005). Ainsi, à l'exception du travail administratif (déclaration PAC, enregistrement des naissances, comptabilité, etc.) et de la prise de décision, ils assurent une grande diversité de tâches parmi lesquelles les soins quotidiens aux bêtes, la traite, la pose et l'entretien des clôtures, le nettoyage des bâtiments, le travail des surfaces et les épandages impliquant la conduite d'engins ou encore les coupes de bois et même parfois de la petite maçonnerie. Alors que le salariat partagé confronte l'employé à une diversité de systèmes techniques et de façons de faire lui offrant ainsi la possibilité de mûrir son projet professionnel et de sécuriser son installation (Madelrieux et al., 2009), le salariat individuel est propice à diversifier encore davantage ses compétences (conduite d'engins en terrain en pente par exemple). La capitalisation des connaissances et l'accumulation, voire la diversification d'expériences, ne profitent pas au seul salarié. Elles bénéficient également au groupement d'employeurs dans la mesure où elles constituent "un bien partagé " (Elyakime, 2007). Si le salarié fait circuler des informations et peut suggérer quelques innovations (toutefois jamais reprises par les employeurs en ce qui concerne ce terrain), ce type d'emploi exige une grande discrétion, prévue par le contrat de travail mais également systématiquement mise en avant spontanément par les salariés.

Parmi l'ensemble des tâches confiées, la traite occupe une place à part. Il s'agit d'un travail astreignant dont la délégation ne va pas de soi (Chabanet et al., 2000). Car pour être biquotidienne, elle n'en est pas moins décisive : c'est un poste à haute responsabilité qui touche au cœur de l'exploitation. La salle de traite donne en effet accès à des informations importantes, qu'il s'agisse de la production laitière proprement dite, de l'état sanitaire du troupeau, de la conformité, de l'entretien et de la propreté des installations. De fait, les réticences au salariat ne relèvent certainement pas des seules raisons économiques: "Y'en a plus d'un qui mettraient pas quelqu'un dans leur salle de traite ", pointe un éleveur-employeur. De plus, astreinte ne signifie pas routine: le salarié doit mobiliser son sens de l'observation, être capable de repérer une bête en chaleur, de diagnostiquer une mammite, de respecter les consignes, ou encore de relayer une information importante. Si en salariat individuel, la traite est souvent constitutive du poste - voire en représente le cœur de métier -, en salariat partagé, sa délégation témoigne d'une certaine confiance de l'employeur et indique qu'une étape est franchie dans l'intégration du salarié et la reconnaissance de ses compétences.

L'image du valet de ferme préposé au "sale boulot " n'est jamais très loin lorsqu'on évoque le salariat agricole et plus encore le travail partagé, particulièrement en service de remplacement. Le "sale boulot " désigne un travail répétitif, fatigant et peu gratifiant fondé sur une tâche d'exécution qui ne mobilise ni compétence, ni savoir-faire ou habileté particuliers, ni capacité d'initiative, tout en reposant sur un outillage rudimentaire (curetage des bâtiments, évacuation manuelle des déchets, nettoyage des installations). La spécialisation d'un salarié dans des tâches d'exécution est une des dérives les plus courantes de ce type d'emploi. De telles situations n'ont pas été observées. On peut avancer deux explications. D'une part, les adhérents d'un même groupement d'employeurs se contrôlent mutuellement de façon d'autant plus efficace que la structure et la zone couverte sont restreintes. D'autre part, il est évident que les conseillers consultés au préalable de l'enquête, nous ont orientés vers des éleveurs honorablement connus en tant qu'employeurs et donc peu enclins à de telles dérives. Mais pour autant, sans y être cantonné, le salarié n'est pas épargné par "le sale boulot ", partie prenante du métier mais qui se partage avec l'employeur.

\section{Avoir plusieurs " patrons "}

L'emploi partagé peut offrir un confort moral au salarié en lui évitant d'être isolé dans une relation salariale éventuellement difficile et lui assurant, grâce à la " triangularisation de l'emploi ", une neutralité en cas de litige dans l'affectation de son temps de travail (Zimmerman, 2006). En contrepartie, le salarié doit être un véritable "caméléon" et faire preuve d'une excellente capacité d'adaptation : "Je fais comme ils font. Je fais pas à mon idée, pour pas chambouler les vaches." Dans tous les cas, et en l'absence du chef d'exploitation, ce sont les vaches les premières et les meilleures juges : "Ca se connaît tout de suite [...]. Le seul juge, c'est le tank à lait!", analyse une agricultrice.

Le vacher de remplacement est susceptible de travailler dans des exploitations qu'il ne connaît pas ou qu'il connaît mal. L'employeur a parfois le temps de lui laisser quelques indications mais pas toujours. De plus, même dans ce cas, les 
situations peuvent vite se compliquer et c'est souvent la traite qui inspire le plus de crainte. "Si y'a vraiment de l'orage, et qu'il y a plus d'électricité... Alors là ! Brancher les groupes électrogènes... Chacun a aussi un machin spécial. C'est pas facile quand les gars ne sont pas là ", reconnaît ce salarié de 46 ans, fort de 13 ans d'ancienneté au sein du même service de remplacement. C'est pour prévenir ce genre de situation que ce service a introduit une nouvelle règle : les adhérents doivent employer le salarié quelques jours hors coups durs, de façon à ce que ce dernier prenne contact avec chaque exploitation où il sera potentiellement appelé à travailler.

Le temps de travail est généralement de 35 heures annualisées, que le salarié "marque [ses] beures, chaque jour et [fasse] signer [sa] feuille" par le chef d'exploitation (autrement dit qu'il " pointe " comme l'ouvrier de l'usine) ou que, au contraire, il se refuse à cet enregistrement écrit du temps de travail qui réaffirme la primauté du contrat et du référent industriel. Les horaires et les rythmes de travail sont particuliers pour les salariés de service de remplacement et de groupement d'employeurs. Ils demandent encore plus de flexibilité par rapport à un emploi permanent au sein d'une exploitation et se prêtent moins à "l'arrangement "dans la mesure où tous les employeurs doivent se concerter. Certaines journées obligent les salariés à de nombreux déplacements. Même si des indemnités sont prévues, il vaut mieux ne pas habiter trop loin de ses employeurs (c'est un cas fréquent d'abandon de poste) et que les exploitations agricoles ne soient pas, quant à elles, trop dispersées. Les vacances sont planifiées en dehors des grosses périodes de travail, selon les termes du contrat de travail, lequel est parfois l'objet de quelques entorses. Et ce n'est pas forcément à la demande de l'employeur car il existe une part d'implicite qui échappe au contrat et contribue à la singularité de la relation salarié/employeur: "Une partie que je pose de vacances, celles-là, j'espère les garder... mais vis-à-vis des agriculteurs, d'un côté, je les connais pratiquement tous, c'est des copains, quoi. C'est vrai qu'à 5-6 ans près, on a le même âge, quoi. C'est sûr que c'est dur de refuser", reconnait le vacher de remplacement.

Dans les situations considérées, l'interconnaissance, la reconnaissance professionnelle réciproque, l'amitié même et le sens du service ont parfois raison de la relation contractuelle qui cède alors la place à des "arrangements " par nature domestiques. Dans ce cas, le salarié reprend à son compte les contraintes (par exemple météorologiques) qui pèsent sur l'organisation du travail agricole. Cette volonté de se "rendre disponible " est compensée de deux façons : selon le registre juridique et marchand, c'est-à-dire en accord avec le contrat de travail (annualisation du temps de travail, majoration des jours fériés, récupération ou paiement des jours supplémentaires), ou selon le registre domestique par des accords interpersonnels oraux relevant d'une réciprocité différée (prêt de matériel, jour libre accordé au pied levé, arrangement d'horaires tel jour, etc.) : " S'il faut donner un coup de collier au foin, ils peuvent compter sur moi. On s'arrange après pour être rétribué, c'est une chose après. [...] Je ne suis pas un ouvrier qui regarde la montre et qui part. [...] Je sais qu'il y a des imprévus et ça, j'accepte. D'un autre côté, ils me le rendent bien." Ce salarié insiste sur le caractère $a$ posteriori de l'arrangement, témoignant de sa confiance dans l'employeur et de son sens du travail accompli. À l'inverse, en cas d'abus dans le partage - inégal - des tâches, il se réserve la possibilité de rester dans la relation salariale strictement prévue par son contrat.

\section{Discussion}

Les premiers éléments dégagés dans cette analyse - qui demande maintenant à être mise à l'épreuve d'un échantillonnage plus important - montrent, dans le contexte étudié, l'importance de la confiance lors du recrutement du salarié. Celle-ci est clairement corrélée à la proximité sociale et/ou professionnelle du candidat. La maîtrise d'un large répertoire de pratiques, renvoyant à des gammes de qualifications mesurables dans l'espace marchand, est requise étant donné la polyvalence de ces emplois. Mais l'habileté et la virtuosité techniques doivent être distinguées d'une autre aptitude fondatrice des métiers de l'élevage, qui consiste à "être bien avec les animaux" (Soriano, 2002). Cette capacité n'est pas liée à la formation scolaire ; elle s'acquiert et se développe " tôt et intimement " : dès l'enfance. Par ailleurs, des qualités personnelles telles que la motivation, le sérieux, l'autonomie, le sens des respon- sabilités (auxquelles s'ajoutent dans le cas des emplois partagés, une forte capacité d'adaptation et une grande discrétion), plus difficiles à évaluer a priori car elles sont éprouvées dans l'exercice du métier, entrent également et largement en jeu. Pour autant, elles ne seraient encore rien si le salarié ne partageait pas avec son employeur des valeurs, un esprit de métier, une éthique, communs. Barlett (1986) a repéré un tel phénomène chez les salariés permanents aux États-Unis exposés à des conditions de travail pourtant difficiles. Selon l'auteur, l'origine fréquemment rurale ou agricole des travailleurs favorise des comportements et des discours sous-tendus par la même " idéologie agrarienne " que celle affichée par les employeurs. De fait, en accord avec une certaine conception du travail d'élevage, le salarié idéal - comme on dirait le gendre ou le fils idéal - doit montrer qu'il est capable, lorsque cela lui semble nécessaire, de s'affranchir du temps contractuel. Cette exigence de flexibilité n'est pas propre à l'agriculture où, comme dans le bâtiment ou l'hôtellerie et la restauration, elle se traduit par des formes d'emplois temporaires spécifiques. Le salarié permanent doit donc pouvoir combiner ces deux aspects du travail agricole: entrer en familiarité avec lieux, bêtes et terres dans une relation de confiance, tout en offrant si cela s'avère nécessaire, la souplesse d'un travailleur saisonnier. Cela suppose qu'il reprenne à son compte les contraintes que font peser les aléas climatiques sur son employeur et dont, en tant que salarié, il devrait être et se sentir dégagé. Une telle disposition favorise une relation employeur/employé bien particulière qui, tout en étant inscrite dans un cadre marchand et juridique, s'en distancie mais ne se dissout pas non plus complètement dans un rapport de nature paternaliste (Lamanthe, 2008). Elle se construit autour de la figure du salarié "aux35-heures-qui-ne-regarde-pas-ses-heures ", c'est-à-dire qui se pose en rupture avec la référence industrielle. C'est dans ces conditions que peut s'établir entre l'employeur et son salarié une relation sinon de parité - lorsqu'un projet d'association est en jeu par exemple ou dans le cas d'un salarié ancien agriculteur - du moins de parrainage lorsque le salarié est jeune et porte un projet d'installation. En effet, ces relations conduisent non seulement à reconnaître la part et la qualité du travail accompli par le salarié, mais aussi, et bien au-delà, à lui attribuer un 
statut de pair et une place au sein d'une famille professionnelle, avant qu'une autre place lui soit faite, peut-être, dans sa propre famille dont il reprendra l'exploitation. On serait alors tenté d'apparenter le salariat au rite de passage tel que défini par Van Gennep (1981), c'est-à-dire un processus social reposant sur une mise à l'écart momentanée d'un individu, suivie de sa réintégration sous un autre statut, plus valorisant (ici, chef d'exploitation). Dans le cas étudié, le salariat contribue à garantir la pérennité du groupe socioprofessionnel; la confrontation de configurations d'élevage et de cultures pastorales pour le moins contrastées (Chine, Sénégal, Brésil) rend compte de la pluralité des formes d'engagement dans l'activité agricole (Madelrieux et al., 2010).

\section{Conclusion}

Quel que soit le cas de figure, le salariat permanent interroge l'évolution du rapport entre structure de parenté et structure de production qui fonde, entre autres critères, la spécificité des exploitations familiales. Le travail des femmes en dehors de l'exploitation caractérise désormais l'agriculture familiale des pays développés, que ce soit en Europe (Djurfeldt et Waldenström, 1996), aux États-Unis (Findeis et al., 2002) ou encore au Canada (Machum, 2005). Ainsi que nous l'avons évoqué en introduction, une telle évolution contribue à accroître la charge de travail du chef d'exploitation, situation à laquelle le recours au salariat remédie en partie. Mais le rapport entre main-d'œuvre familiale et emploi non familial permanent ne s'opère pas nécessairement toujours selon cet agencement. Ainsi, dans les zones très rurales d'Écosse, la charge de travail augmente en raison de l'agrandissement des structures et du départ précoce, vers la ville, des enfants du ménage, par ailleurs souvent fort peu enclins à s'investir dans l'exploitation. Or la possibilité de recruter un salarié est très limitée : le marché local de l'emploi n'est pas favorable car les jeunes quittent précocement le pays et le coût économique est fortement dissuasif. On est donc dans une situation totalement inverse à celle rencontrée en France : ce sont les épou- ses qui prennent en charge le travail excédentaire, et s'engagent donc dans des tâches agricoles qui ne leur sont pas traditionnellement dévolues (Burton et al., 2005). De tels contrastes illustrent la complexité des facteurs qui déterminent plus ou moins fortement la réorganisation du travail dans les exploitations agricoles et favorisent - ou non - le développement d'un salariat permanent. Ils pointent la nécessité de prendre en compte un ensemble de dimensions économiques, démographiques, juridiques, sociales, mais aussi largement culturelles. Enfin, s'il est fécond de raisonner en termes de substitution de la main-d'œuvre familiale par le salariat permanent, l'existence d'un contrat de travail marque une des limites d'un tel cadre d'analyse. Ce document établit nettement (et rappelle lorsque cela s'avère nécessaire) la différence entre travail salarié et travail familial. À ce titre, le rapport souvent conflictuel à la flexibilité apparaît comme une des questions saillantes. Dans un univers familial de production, la disponibilité de chacun relève d'une norme établie. À défaut d'avoir été choisie par tous, elle est consentie par chacun des membres du collectif et parfois entre plusieurs générations (Wallace et al., 1994; Melberg, 2005). En revanche, dans le cadre d'un emploi contractuel, la flexibilité est encadrée par une règle de droit qui, tout au moins dans les textes, protège le salarié de certaines dérives. En effet, le contrat tient l'employé à distance du groupe domestique de l'employeur tout en l'affiliant à une famille professionnelle indépendante de toute structure de parenté.

\section{Remerciements}

Ces travaux ont bénéficié du soutien financier de l'Agence nationale de la recherche (programme Agriculture et développement durable: projet ANR05-PADD-003 TRANS)

\section{Références}

Barthez A. Famille, travail et agriculture. Paris: Economica, 1982.

Barlett PF. Profile of full-time farm workers in a Georgia county. Rural Socio 1986 ; 51 : 78-9.
Bourquelot F. Les salariés agricoles en France. Paris : École Pratique des Hautes Études, 1973.

Burton R, Schwarz G, Fischer H. Main-d'œuvre agricole et plan de développement rural en Écosse. Economie Rurale 2005; (289-290): 106-29.

Chabanet G, Dedieu B, Servière G, Tchakérian E. Le salariat partagé : caractéristiques et fonctionnement des groupements en région d'élevage d'Auvergne et du Limousin. Cah Agric $2000 ; 9$ : 23-8.

Djurfeldt G, Waldenström C. Towards a theoretically grounded typology of farms: A Swedish case. Acta Sociol 1996 ; 39 : 187-210.

Elyakime B. Groupement d'employeurs agricoles: quelle aide publique locale? Revue d'Economie Régionale \& Urbaine 2007 ; 5 : 861-80

Frappat B. Salariat en élevage laitier : des élèves, des salariés et des éleveurs nous donnent leur vision. Compte-rendu final de l'étude. Collection Etudes. Paris: Institut de I'élevage, 2005. www. inst-elevage.asso.fr/htm/1/IMG/pdf/synthese_ salariat_CIL_07-06.pdf

Findeis JL. Hired farm labour Adjustments and constraints. In : JL, VandemanAM, Larson JM, Runyan JL, eds. The dynamics of hired farm labour. Constraints and community responses. Wallingford (Great Britain): CABI Publishing, 2002.

Lamanthe A. Les paradoxes de la relation salariale en milieu rural (Briançonnais). Etudes Rurales $2008 ; 182$ : 29-44.

Madelrieux S, Dupré L, Rémy J. Itinéraires croisés et relations entre éleveurs et salariés dans les Alpes du Nord. Economie Rurale 2009; 313-314: 6-21.

Madelrieux S, Hostiou N, Dupré L, et al. Liens entre salariat et activité agricole: itinéraires professionnels de salariés d'élevage. Cah Agric $2010 ; 19$ : epub. doi : 10.1684/agr.2010.0426.

Maget M. Remarques sur le village comme cadre de recherches anthropologiques. Bull Psycho $1955 ; 8: 373-82$.

Machum S. The persistent of Family farming in the wake of Agribusiness: A new Brunswick, Canada case study. J Comp Fam Stud 2005; 26 : 377-90.

Melberg K. Family farm transactions in Norway : Unpaid care across three generations. J Comp Fam Stud $2005 ; 26$ : 419-42.

Peltier C, Marguet J, Privat C, Coulombel A. La place du travail salarié dans la gestion des exploitations agricoles. Notes et Études SocioÉconomiques 2009 ; 32 : 41-60.

Rattin S. Recrudescence du temps partiel chez les salariés permanents non familiaux. Agreste Cahiers $2003 ; 3: 9-15$.

Soriano V. Étre bien avec les animaux, ça s'apprend? Ethnozootechnie 2002; 68: 85-91.

Van Gennep A. Les rites de passage: étude systématique des rites. Paris: E. Nourry, 1981 [1909].

Wallace C, Dunkerley D, Cheal B, Warren M Young people and the division of labour in farming families. Sociol Rev 1994 ; 42 : 501-30. 